Pirineos, 147-148: 61 a 80, JACA; 1996

\title{
RESULTADOS PRELIMINARES OBTENIDOS A PARTIR DE UNA TRAMPA MALAISE SITUADA EN UNA ZONA MEDITERRANEA PIRENAICA
}

\author{
J. Pujade Villar
}

Universitat de Barcelona. Facultat de Biologia. Departament de Biologia Animal. Unitat d'Artròpodes. Avda. Diagonal, 645. E-08028 Barcelona.

RESUMEN.- En este estudio se da cuenta de los distintos órdenes de artrópodos capturados mediante una trampa Malaise en Santa Coloma (Andorra) después de un año de muestreo. Han sido colectados 141.522 ejemplares pertenecientes a 20 órdenes distintos. El 99,5\% corresponden a insectos, entre los cuales los dípteros (con el $74,1 \%)$, juntamente con los himenópteros $(15,2 \%)$ y los homópteros $(5,8 \%)$ constituyen más del $95 \%$ de las capturas realizadas. Se discute la fenología anual de los distintos grupos en el área de estudio y su relación con las circunstancias meteorológicas del año 1993. En general, las mayores abundancias de los principales grupos se registran entre la segunda mitad de la primavera y la primera mitad del verano debido a las condiciones xerotérmicas de dicha localidad.

ABSTRACT.- In this article data about the several Orders of arthropods captured using a Malaise trap, at Santa Coloma (Andorra), after a one-year cycle is reported. Up to 141.522 specimens have been collected, belonging to 20 different Orders. About $99.5 \%$ of the captures are insects, of which $74.1 \%$ are Diptera, $15.2 \%$ are Hymenoptera and $5.8 \%$ are Homoptera. These three groups together represent $95 \%$ of the arthropods. Annual phenology of the different groups and their relationship with the weather is discussed. Generally, the peaks of abundance of the main groups are registerd in the second stage of the spring and the first one of the summer. It is due to the xerothermic conditions of the locality.

RÉSUMÉ.- On montre les premiers résultats obtenuis après avoir mis une piège Malaise pendant une année à Santa Coloma (Andorra). Nous avons collecté un total de 141.522 exemplaires qui appartiennent à 20 ordres différents d'arthropodes. Le 99,5\% des captures sont des insectes, les diptères (avec le $74,1 \%$ ), en meme temps que les hyménoptères (avec le 15,2\%) et les homoptères (5,8\%) représentent plus du $95 \%$ des captures. On parle de la phénologie annuel des différents groupes capturés et de leur relation avec les conditions météorologiques de 1993. En général, les plus grandes abondances des principaux groupes ont été enregistrées pendant la seconde moitié du printemps jusqu'à la première moitié de l'été, dî àles conditions xérothermiques de cette localité. 
Keywords: Malaise trap, Arthropoda, Insecta, Collembola, Arachnida, Andorra.

\section{Introducción}

La trampa Malaise es el sistema pasivo de captura entomológica más eficaz atendiendo al número de ejemplares colectados. No obstante, se muestra claramente selectiva, puesto que el mayor número de capturas corresponden a dípteros; éstos, juntamente con los himenópteros, constituyen el $90 \%$ del total de ejemplares estudiados. El material, que es colectado en alcohol, un medio idóneo de conservación para la mayoría de los artrópodos, se obtiene en perfecto estado. Si además tenemos en cuenta que actúa ininterrumpidamente día y noche, todo ello hace que este método pueda ser un buen modelo a utilizar tanto para estudios cuantitativos como cualitativos.

Por lo que hace referencia a los estudios cuantitativos, cabe destacar, por nombrar algunos, los realizados por Marston (véase STEYSKAL, 1981 y MATHEWS \& MATHEWS, 1970 en Estados Unidos), por Moczar en Hungría (véase STEYSKAL, l.c.), por KRZELJ (1969) en Francia, por TERESHKIN \& SHLYAKHTYONOK (1989) en Rusia y NIEVES-ALDREY \& REY del CASTILLO (1991) en España, todos ellos exponiendo la trampa dentro de un intervalo inferior al anual (Tabla I). Los resultados cuantitativos desprendidos después de una exposición anual de la trampa Malaise se reflejan por ejemplo en GeiJkes en Surinam (ver STEYSKAL, 1981 y más recientemente en NIEVES-ALDREY (com. pers.) en España. El estudio que se presenta es por tanto una nueva aportación al conocimiento de la fluctuación de algunos grupos de artrópodos en el período anual en un punto determinado. Por otra parte, también podemos encontrar estudios cuantitativos referidos a órdenes concretos de insectos; es el caso, por lo que hace referencia a los himenópteros, de NOYES (1989a y b), DARLING \& PACKER (1988), TAN (1990), TAN \& al. (1990) y NIEVES-ALDREY \& REY del CASTİLLO (1991).

Los estudios cualitativos son mucho más lentos y requieren el concurso de especialistas en los distintos grupos colectados. A pesar del numeroso material obtenido en distintos puntos de la Península Ibérica (más de un millón y medio de ejemplares), son aún muy escasas las referencias taxonómicas referidas. Cabe destacar que parte de dicho material está aún en proceso de estudio. No obstante, han sido publicados datos parciales por ASKEW (1991, 1993 y 1994) referentes a los calcidoideos (Hymenoptera) de Pina de Ebro (Zaragoza), CARLES-TOLRÁ (1992a, 1992b, 1993a y b) referidos a Trixoscelídidos, Helomícidos y Cárnidos (Diptera) de Pina de Ebro 


\begin{tabular}{|l|c|c|c|c|c|c|c|c|c|c|}
\hline & $\begin{array}{c}\text { EEUU } \\
(1964)\end{array}$ & $\begin{array}{c}\text { Hungría } \\
(1966)\end{array}$ & $\begin{array}{c}\text { Surinam } \\
(1965-66)\end{array}$ & $\begin{array}{c}\text { Francia } \\
(1967)\end{array}$ & $\begin{array}{c}\text { EEUU } \\
(1967)\end{array}$ & $\begin{array}{c}\text { Rusia } \\
(1985)\end{array}$ & $\begin{array}{c}\text { España } \\
(1988)\end{array}$ & $\begin{array}{c}\text { España }(1) \\
(1989-90)\end{array}$ & $\begin{array}{c}\text { España (2) } \\
(1991-92)\end{array}$ & $\begin{array}{c}\text { Andorra } \\
(1993)\end{array}$ \\
\hline Apterigota & - & - & - & - & - & - & - & $*$ & $*$ & - \\
\hline Odonata & 0,1 & 0,2 & $*$ & - & $*$ & - & - & - & - & - \\
\hline Ephemeroptera & - & $*$ & - & - & $*$ & - & $*$ & $*$ & - & $*$ \\
\hline Plecoptera & - & - & - & - & 20,8 & - & 0,4 & 0,15 & - & $*$ \\
\hline Orthoptera & 0,5 & $*$ & 1,7 & 0,3 & $*$ & $*$ & 0,2 & $*$ & 0,05 & 0,05 \\
\hline Dictyoptera (s.l.) & - & - & - & 0,2 & - & - & 0,05 & $*$ & $*$ & $*$ \\
\hline Dermaptera & - & - & - & 0,1 & - & 0,4 & 0,05 & $*$ & 0,13 & 0,06 \\
\hline Psocoptera & - & - & 0,1 & - & 0,2 & - & $*$ & $*$ & 0,05 & 0,14 \\
\hline Thysanoptera & - & - & - & - & 0,9 & 1 & 0,4 & 0,37 & 0,16 & 0,2 \\
\hline Hemiptera (s.l.) & 6,8 & 5,8 & 2,3 & 0,8 & 7,1 & 2,3 & 4,2 & 3,84 & 2,16 & 6,39 \\
\hline Diptera & 66,3 & 79,8 & 58 & 73,4 & 44,5 & 59 & 71,4 & 80,76 & 73,02 & 74,15 \\
\hline Trichoptera & - & $*$ & - & - & 0,7 & - & $*$ & $*$ & - & $*$ \\
\hline Lepidoptera & 4,8 & 3 & 14 & 4,8 & 7,2 & 12 & 2,06 & 1,28 & 4,54 & 2,02 \\
\hline Mecoptera & - & 0,2 & - & - & $*$ & $*$ & - & $*$ & - & $*$ \\
\hline Neuroptera (s.l.) & 0,1 & $*$ & 0,4 & - & 0,1 & 0,2 & 0,07 & 0,05 & 0,1 & 0,22 \\
\hline Coleoptera & 5,6 & 0,3 & 4,6 & 3,2 & 2,4 & 2 & 3,05 & 1,78 & 1,26 & 1,33 \\
\hline Strepsiptera & - & - & - & - & - & - & - & - & - \\
\hline Hymenoptera & 12,3 & 10,7 & 19 & 16,8 & 14,7 & 23 & 17,8 & 11,61 & 18,43 & 15,28 \\
\hline Total ejemplares & 2.027 & 20.713 & 90.182 & 10.495 & 40.348 & 34.174 & 49.386 & 531.237 & 170.818 & 140.694 \\
\hline Ej. colect. en igual \\
período en Andorra
\end{tabular}

* presencia, aunque inferior al 0,05\% $\quad$ (1) y (2) Datos cedidos por J. L. Nieves-Aldrey (CSIC)

Tabla I. Comparación de los porcentajes de los órdenes de insectos obtenidos en distintos muestreos con trampa Malaise, a partir de los datos proporcionados por NIEVES-ALDREY \& REY del CASTILLO (1991)

Comparison of the percentages of insect orders collected using Malaise traps. Data from NIEVES-ALDREY \& REY del CASTILLO (1991). 
(Zaragoza), DELÉCOLLE \& RIEB (1993) en relación a los Ceratopogónidos (Diptera) colectados en Pina de Ebro (Zaragoza), GARRIDO-TORRES \& NIEVES-ALDREY (1992a) por lo que hace a los Pteromálidos (Hymenoptera) colectados en San Pedro de Bedoya, Aldea de Pinar y Villanueva de Pría (Santander, Burgos y Oviedo, respectivamente, España), GARRIDOTORRES \& NIEVES-ALDREY (1992b) en referencia a los Pteromálidos (Hymenoptera) de El Ventorrillo (Madrid), MOHRING \& BLASCO-ZUMETA (1992) en relación a los Simúlidos (Diptera) de Pina de Ebro (Zaragoza), GEIGER (1993) en relación a los Limonídos (Diptera) de Pina de Ebro (Zaragoza, España), y GESSÉ, \& al. (1994) en atención a los heterópteros colectados en Santa Coloma (Andorra).

Cabe advertir que la trampa Malaise muestrea únicamente insectos voladores; el resto de capturas pueden considerarse como accidentales ya que la trampa no se diseñó para estos grupos. Además las capturas están en relación directa con dos parámetros: la abundancia y la movilidad; ésta última depende a su vez de la temperatura. Por ello, ha de quedar claro que las conclusiones que se extraen se basan en frecuencias de capturas, en ningún caso en densidades, y por tanto estos datos sólo serán válidos para comparar con otros de trampas iguales.

\section{Material y método}

La trampa Malaise empleada corresponde al modelo Townes (de origen comercial Marris House Nets, London), de malla fina y de color negro.

El material en que se basa el presente trabajo es el colectado durante todo 1993 ininterrumpidamente. Las muestras fueron recogidas con una periodicidad de dos veces al mes de las que la primera muestra mensual se corresponde a la primera quïncena del mismo y la segunda al período restante (oscilando por tanto las segundas muestras entre 13 y’16 días según la mensualidad). El medio colector que se empleó fue etanol al $80 \%$.

En la ubicación de la trampa se tuvo en cuenta no sólo la orografía del terreno (pues en su zona posterior se presenta la base del valle del Roc de Sant Vicenç por lo que es una área de viento direccional y en su lateral derecho un roquedo de piedra caliza de unos 30 metros de altura que puede actuar como barrera natural), sino también la localización de las manchas de bosque cercanas a la zona de estudio. Por todo, ello la trampa fue instalada en el espacio abierto entre una mancha densa de vegetación y el muro calizo. El recipiente colector fue dirigido hacia la zona abierta de mayor luminosidad (expuesta al Norte), la parte posterior se encontraba lateralmente respecto a la desembocadura del Valle. 


\section{3. Área de estudio}

La trampa utilizada estuvo instalada en Santa Coloma (Andorra), en la Vall del Roc de Sant Vicenç, cerca del río de Enclar, en el terreno particular de Can Miqueldolça, a $1.050 \mathrm{~m}$ de altitud. El terreno es kárstico y corresponde al límite del entorno mediterráneo, en el que el encinar (Quercus ilex), degradado por la altitud, se encuentra parcialmente sustituido por pies vigorosos de roble pubescente (Quercus humilis). Por su situación, encontramos una mezcla de vegetación propia de la alianza Quercion ilicis con elementos de Quercion pubescentipetraeae (por ser una zona de transición entre dichas comunidades), en la que se observa además la presencia de pies pertenecientes a plantas cultivadas venidas de zonas adyacentes junto con elementos propios de ribera, pues tanto el róo Valira como alguno de sus afluentes circundan la zona de muestreo. Así, atendiendo al estrato arbóreo, además de los ya mencionados, también destacan pies más o menos dispersos de roble albar (Quercus petraea), castaño (Castanea sativa), temblón (Populus tremuula), pino albar (Pinus sylvestris), avellano (Corylus avellana), cerezo (Prunus avium), laurel (Laurus nobilis) y sauquillo (Sambucus ebulus). En el sotobosque destaca como liana la hiedra (Hedera helix); la zarza (Rubus sp), los rosales (Rosa spp.) y el boj (Buxus sempervirens) son los arbustos principales, si bien podemos encontrar enebro (Juniperus commmunis), sabina (Juniperus phoenicea) y acebo (Ilex aquifolium). En el estrato herbáceo en la zona la jabonera (Saponaria officinalis), diversos tréboles (Trifoliunn spp.), gramíneas de diversos géneros, ortigas (Urtica dioica), diente de león (Taraxacum officinale), gordolobo (Verbascum thapsus), eléboro verde (Helleborus viridis), clavelina (Dianthus sp), helechos como Pteridium aquilinum, siempreviva (Helicrysum stoechas), alfalfa (Medicago sativa) y tabaco (Nicotiana tabacum), entre muchas otras.

Atendiendo a la peculiaridad del relieve de la zona, dicho valle es un lugar de transición entre enclaves mediterráneos y dominios medioeuropeos o boreoalpinos que están situados a mayor altitud.

La climatología de la zona es variable, si bien se corresponde al modelo mediterráneo a grandes rasgos, bajo la influencia de altas presiones subtropicales. El tiempo no sólo depende de las situaciones dominantes sino también de la orografía propia de la zona, que en el caso de los Pirineos ejerce una incidencia decisiva sobre las condiciones atmosféricas. Lá precipitación anual depende en gran parte del agua caída durante los meses de mayor temperatura aunque el número de días de precipitación sea claramente superior en el otoño y sobre todo en la primavera, en relación al verano (RASO-NADAL, 1992).

Los datos de pluviosidad y temperatura han sido cedidos por la estación meteorológica del «Lycée Comtes de Foix», observatorio situado cerca del área de estudio a $1.000 \mathrm{~m}$ de altitud (Fig. 1). 


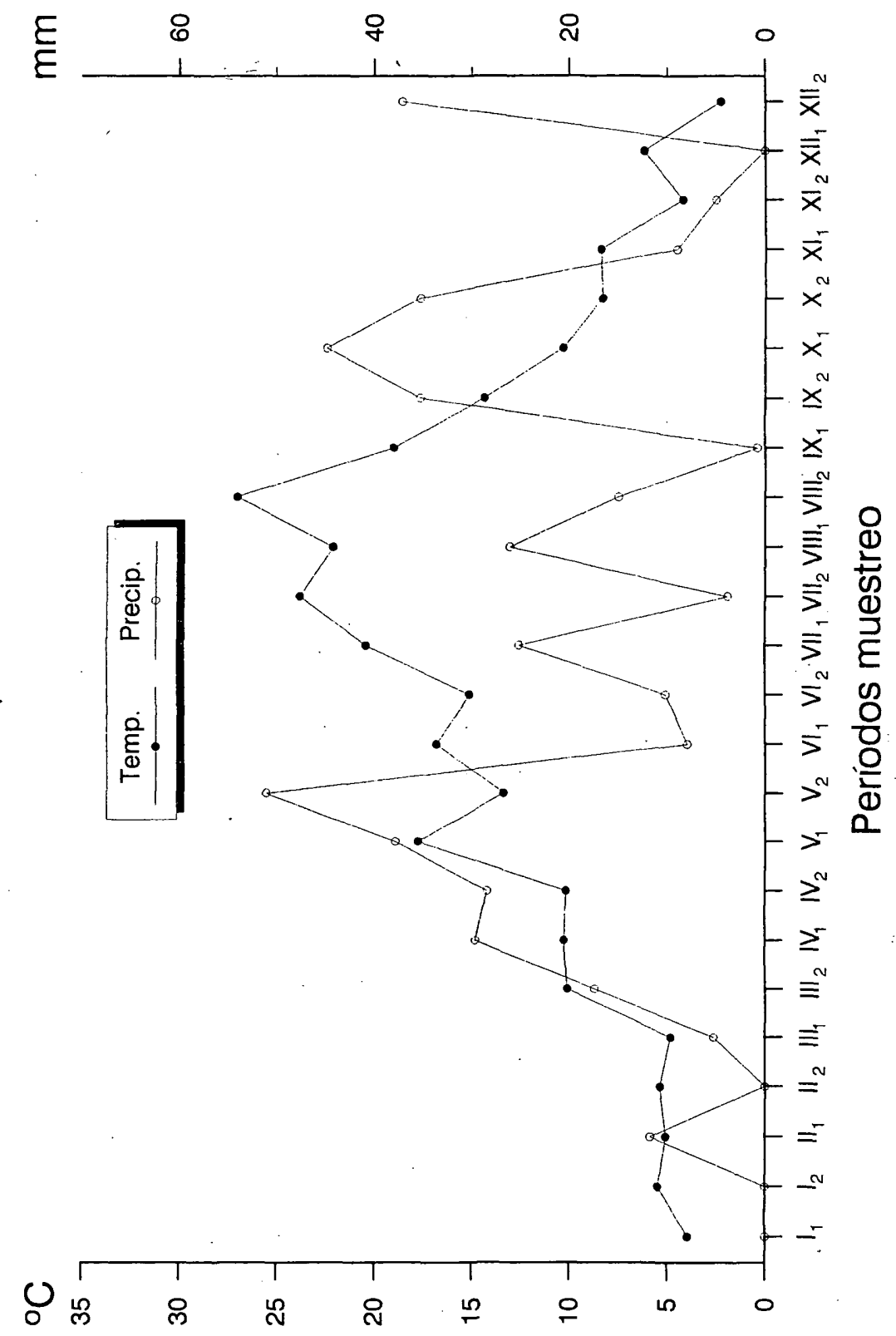

Fig. 1. Diagrama bioclimático de la zona estudiada para cada período muestreado. Bioclimatic diagram of the studied area for each sampled period. 
El año de estudio se caracterizó por ser fundamentalmente seco en comparación con el año anterior; así, se recogieron en 1993 un total de 445,4 1/ $\mathrm{m}^{2}$ mientras que en 1992 el valor ascendió a 973,4 1/ $\mathrm{m}^{2}$ (lo cual significa, una diferencia de $5281 / \mathrm{m}^{2}$, más de toda el agua caída durante todo 1993). Atendiendo a la temperatura hemos de mencionar varias cosas. En primer lugar, que durante más de tres meses no se superan $\operatorname{los} 5^{\circ} \mathrm{C}$ de temperatura media y que durante 8 meses la temperatura media no alcanza los $15^{\circ} \mathrm{C}$, ello hace que la proliferación de los artrópodos se vea restringida a un período de tiempo relativamente corto. En segundo lugar, las fluctuaciones térmicas día/noche en un intervalo mensual corto (o incluso las fluctuaciones día a día) oscilan o pueden oscilar muchísimo (Fig. 2). En tercer lugar, los intervalos de temperatura máximos y mínimos anuales, en la zona de estudio, se mueven en un intervalo muy amplio (desde $36,6^{\circ} \mathrm{C}$ hasta $-9^{\circ} \mathrm{C}$, en el período muestreado). Todo ello ejercerá mayor o menor influencia en la presencia de los distintos grupos de artrópodos.

\section{Resultados}

Los resultados excluyen tan sólo las larvas de insectos holometábolos de forma que se incluyen los estados inmaduros de los heterometábolos, todos los insectos adultos (alados, braquípteros y ápteros primarios o bien secundariamente ápteros), los colémbolos y los arácnidos.

El total de ejemplares colectados es de 141.522 (Tabla II), de los que el $99,51 \%$ son insectos pertenecientes a 20 órdenes distintos. En la figura 3 se representa gráficamente la composición porcentual de los distintos órdenes. Se observa que el componente mayoritario es sin duda el de los dípteros que constituye más del $74 \%$ de capturas. Estos, juntamente con los himenópteros (con el 15,2\%) y los homópteros (con el 5,8\%), constituyen el 95\% de las capturas de insectos. El resto de órdenes alcanzan representaciones mucho mas bajas, superando el $1 \%$ tan sólo los coleópteros y lepidópteros.

Los datos referidos a las capturas realizadas en cada período de muestreo (fig. 4) indican la estacionalidad característica de la zona de estudio. En efecto, a partir de la primera quincena de mayo se observa una duplicación de las capturas realizadas respecto a las quincenas del mes anterior. Por otro ladó durante la segunda quincena del mes de mayo y durante todo junio (que se corresponde con la mensualidad más prolífica) se obtiene más de la tercera parte del total del material colectado durante el año completo. Si a estos datos además les sumamos las capturas de agosto el valor obtenido se acerca al 60\% de las capturas totales. Es interesante destacar el desplazamiento hacia la primavera que sufre la población debido a la relativa rigidez climá- 


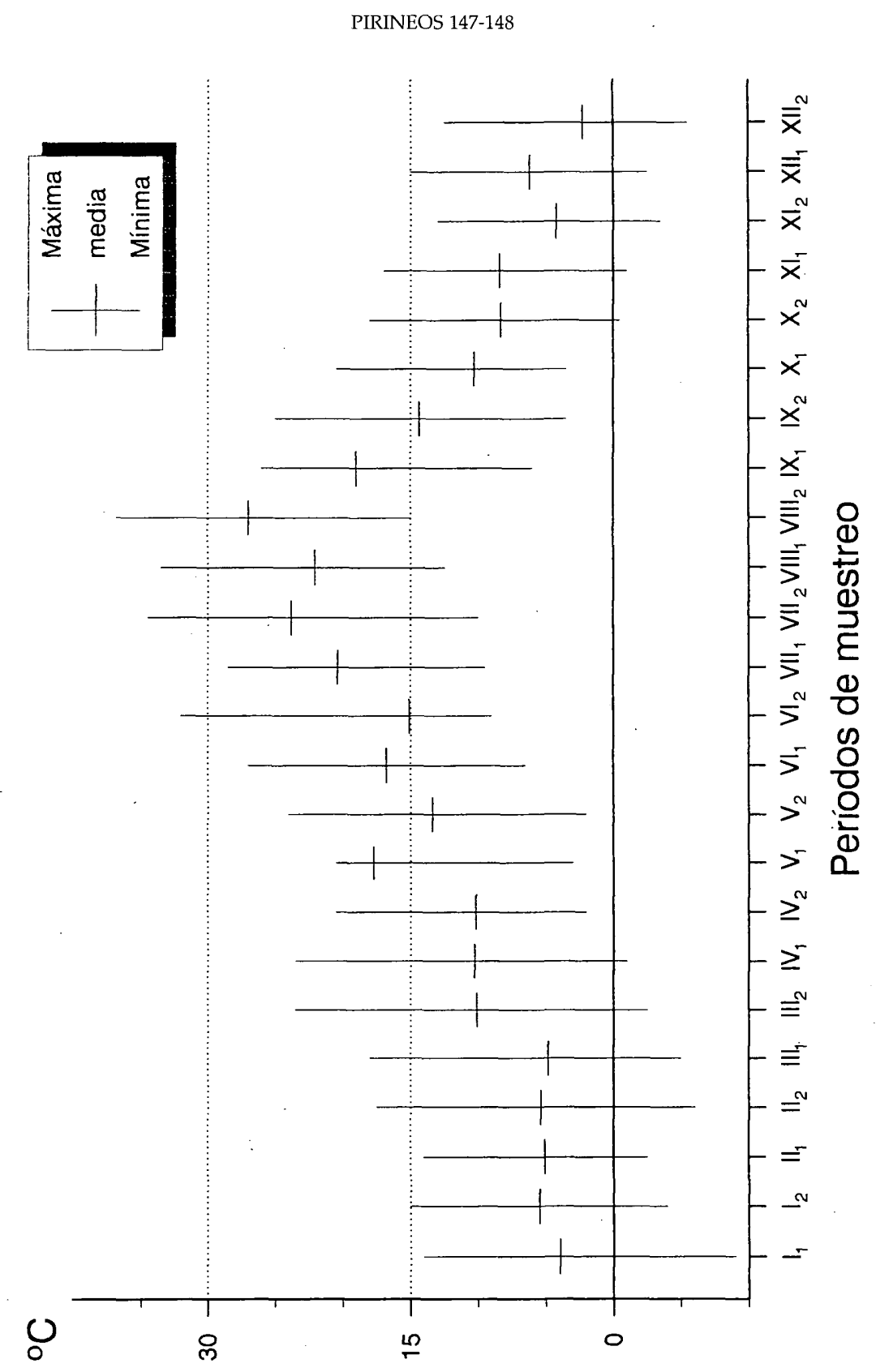

Fig. 2. Oscilaciones térmicas de los distintos períodos de muestreo. Thermic oscillations in the periods of sample. 


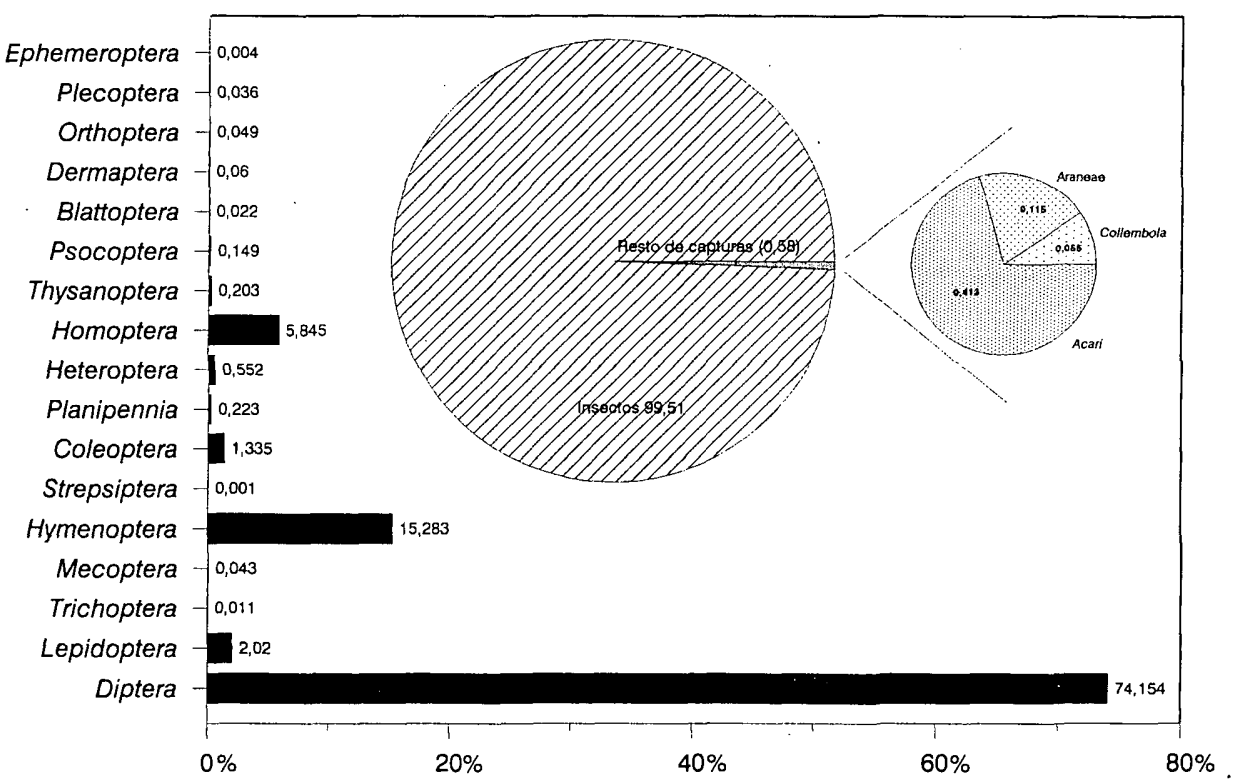

Fig. 3. Porcentajes de los distintos órdenes sobre el total de ejemplares colectados.

Percentages of the different orders of insects in relation to the total number of specimens collected.

tica de la zona estudiada. Por ello podemos observar que a finales de primavera y durante el primer tercio del verano la población es máxima, disminuyendo visiblemente durante la segunda parte del verano y siendo ya baja la población otoñal. Estas variaciones poblacionales de los artrópodos pueden haberse visto acentuadas si tenemos en cuenta la climatología de la zona durante el período de muestreo, aunque como ya hemos mencionado podemos considerar 1993 como un año poco lluvioso. Se observa que a partir de la segunda quincena de junio (fig. 1) se produce una radical disminución de la pluviosidad, la cual vuelve a aumentar al final del estío. A pesar de ello, también debemos hacer notar que, pese a ser la segunda quincena de julio menos lluviosa que la primera y pese a presentar características térmicas en principio más favorables, la captura de ejemplares no es significativamente distinta a la obtenida durante la primera quincena.

Por otro lado la eficiencia de captura, considerada como el número de individuos colectados cada día del período de muestreo es muy variable como era de esperar, oscila entre 38 y 1.408 individuos diarios. Es de resaltar que pese a la rigidez climática son varios los órdenes que presentan actividad durante el período invernal, siendo los dípteros los más abundantes con diferencia (Tabla Il y Figura 4). 


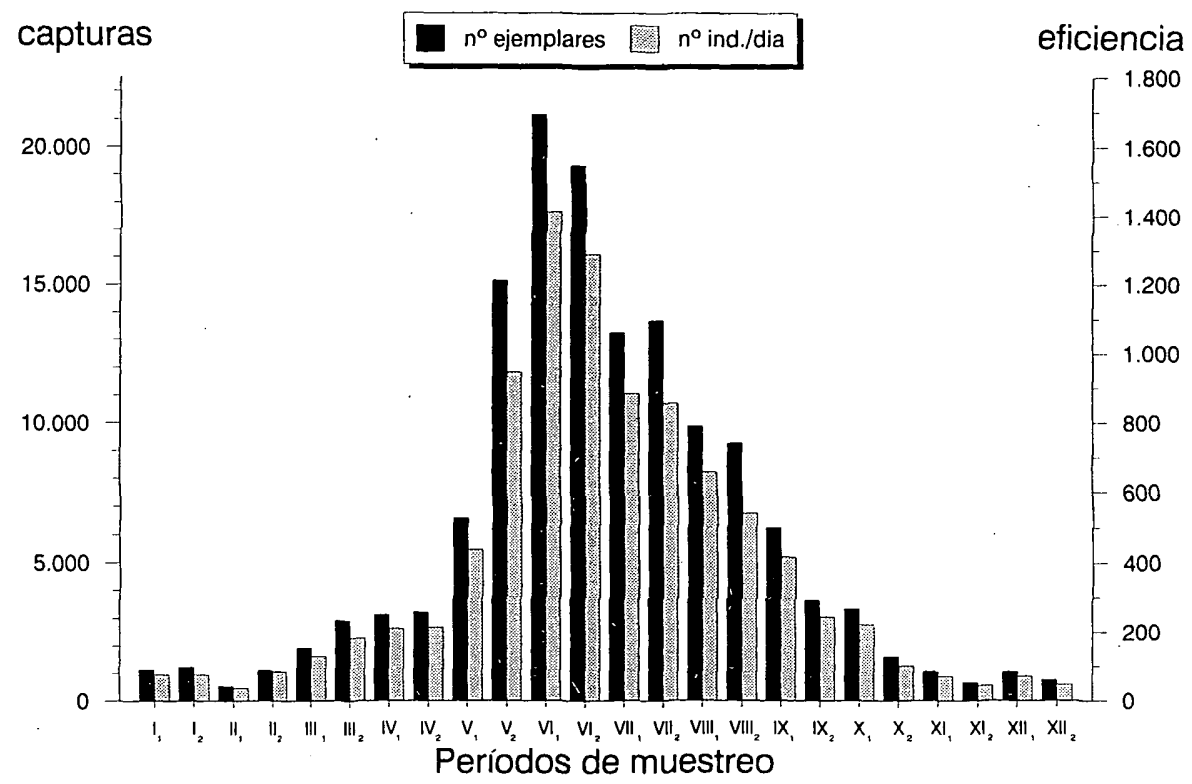

Fig. 4. Evolución de las cifras totales de ejemplares colectados y de la eficiencia de la captura en los distintos períodos de muestreo.

Evolution of the total figures of collected specimens and the efficiency of the capture in the sampled periods.

Es interesante tener en cuenta las cifras relativas referidas a las capturas, no sólo porque nos muestran los períodos de aparición-desaparición de los distintos grupos colectados sino porque también podemos extraer ciertas conclusiones referidas a su fenología particular (teniendo en cuenta ciertas consideraciones que serán abordadas en cada caso concreto).

Atendiendo a las frecuencias relativas de aquellos órdenes mayoritarios '(cuya proporción supera el 5\%) hemos de destacar (Fig. 5) varias cosas. En primer lugar, los dípteros son, con diferencia, el orden mayoritario en todas las quincenas anuales, oscilando su presencia entre el 57,7 y $94,5 \%$. A su vez durante el período invernal se consigue el mayor porcentaje de capturas de dípteros (siempre superior al 87\%), que no es debido al aumento de éstos sino a la disminución o extinción de la presencia del resto de grupos. Aunque el alcohol es en principio un atrayente respecto a los dípteros, la ubicación de esta sustancia en la trampa Malaise no explica que sea la causa por la cual es tan exitoso este modelo de trampeo respecto a este orden, posiblemente ello esté relacionado con un microclima formado en el interior de la misma en el que se ven envueltos los artrópodos en general y los dípteros en particular, al 


\begin{tabular}{|c|c|c|c|c|c|c|c|c|c|c|c|c|c|c|c|c|c|c|c|c|c|c|c|c|c|}
\hline & 1-15.1. & $16-3.1 .1$ & 1-15.1.II & $16-28 . \mathrm{II}$ & 1-1-55.III & 16-3.1.1II & 1-15.5. IV & $\mid 16-30.1 \mathrm{~V}$ & 1-15.V & $15-31 . \mathrm{V}$ & $1-15 . \mathrm{VI}$ & $16-30.0 \mathrm{VI}$ & $1-15 . \mathrm{VII}$ & $|16-31 . \mathrm{VI}|$ & $1-155 . \mathrm{VIII} / 1$ & $|16-31 . V \mathrm{VII}|$ & 1-15.IX & $|16-30.1 x|$ & 1-1-15.X & $16-31 . X$ & 1-15.XI & 16-30.XI & $1-15$. XII & $\mid 16-31 . X I 1$ & I) TOTAL \\
\hline Ephemeroptera & & & & & & & & 1 & & 1 & 3 & 1 & & & & & & & & & & & & & 7 \\
\hline Plecoptera & & & & & & & & & & 6 & 18 & 19 & 2 & & & & & & 1. & & 2 & 2 & 1 & & 51 \\
\hline Orthoptera & & & & & & & & & & 4 & 0 & 6 & 11 & 10 & 11 & 19 & 3 & 3 & 2 & & & & & & 70 \\
\hline Dermaptera & & & & & & & & & & 1 & 1 & 9 & 15 & 20 & 22 & 11 & 4 & & 1 & 1 & & & & & 85 \\
\hline Blattoptera & & & & & & 1 & & & 3 & 10 & 5 & 5 & 3 & 1 & 2 & 1 & & 1 & & & & & & & 32 \\
\hline Psocoptera & 3 & & 1 & & & & & & & 2 & 9 & 10 & 17 & 59 & 41 & 20 & 8 & 13 & 4 & 4 & 1 & & 1 & 2 & 195 \\
\hline Thysanoptera & & & & & 1 & 1 & 10 & 11 & 6 & 15 & 68 & 49 & 51 & 40 & 19 & 14 & & 1 & & & 1 & & & & 287 \\
\hline Homoptera & 21 & 17 & 12 & 40 & 62 & 140 & 175 & 153 & 308 & 666 & 1654 & 1529 & 736 & 542 & 573 & 602 & 325 & 197 & 182 & 123 & 99 & 22 & 41 & 5 & 8224 \\
\hline Heteroptera & & 2 & & 3 & 2 & 4 & 3 & 1 & 3 & 351 & 190 & 56 & 42 & 43 & 31 & 19 & 5 & 7 & 2 & 5 & 2 & 5 & 2 & & 778 \\
\hline Planipennia & 1 & 1 & & 1 & & 10 & 4 & 3 & 11 & 14 & 24 & 49 & 31 & 41 & 62 & 49 & 24 & 7 & 1 & 5 & & & & & 338 \\
\hline Coleoptera & 17 & 4 & 1 & 3 & 13 & 22 & 27 & 10 & 57 & 123 & 150 & 356 & 248 & 343 & 216 & 143 & 29 & 630 & 22 & 25 & 15 & 5 & 21 & 3 & 1879 \\
\hline Strepsiptera & & & & & & & & & & & & 1 & & & 1 & & & & & & & & & & 2 \\
\hline Hymenoptera & 40 & 10 & 9 & 16 & 28 & 138 & 147 & 129 & 462 & 1264 & 2282 & 3288 & 3044 & 3140 & 2592 & 2368 & 1291 & 630 & 237 & 164 & 131 & 24 & 43 & 26 & 21503 \\
\hline Mecoptera & & & & & & & & & 3 & 16 & 17 & 8 & 8 & 4 & 3 & 2 & & & & & & & & & 61 \\
\hline Trichoptera & & & & & & & & & & 2 & 2 & & 1 & & & & 2 & & 1 & & & & & & 8 \\
\hline Lepidoptera & 5 & 6 & 2 & 15 & 9 & 25 & 22 & 15 & 37 & 114 & 203 & 393 & 296 & 422 & 559 & 474 & 101 & 60 & 32 & 11 & 10 & 16 & 15 & 1 & 2843 \\
\hline Diptera & 1042 & 1159 & 495 & 1011 & 1795 & 2552 & 2721 & 2857 & 5657 & 12482 & 16383 & 13416 & 8559 & 8962 & 5689 & 5504 & 4411 & 2662 & 2801 & 1236 & 770 & 557 & 915 & 695 & 104331 \\
\hline Collembola & 2 & 2 & & 2 & & 5 & & 4 & 3 & 5 & 3 & 9 & 6 & 2 & 5 & 4 & 3 & 5 & 7 & 4 & 4 & ${ }^{4}$ & 3 & 1 & 79 \\
\hline Araneae & 1 & 4 & 4 & 3 & 1 & 3 & 4 & 1 & 5 & 14 & 10 & 21 & 9 & 11 & 11 & 10 & 8 & 5 & 11 & 6 & 8 & 5 & 8 & 1 & 164 \\
\hline Acarl & & & & & 2 & 3 & 8 & 17 & 27 & 38 & 107 & 85 & 75 & 49 & 29 & 30 & 26 & 27 & 19 & 8 & 12 & 4 & 4 & 14 & 585 \\
\hline TOTAL COL. & 1132 & 1205 & 524 & 1095 & 1913 & 2904 & 3121 & 3202 & 6582 & 15128 & 21130 & 19310 & 13154 & 13689 & 9866 & 9270 & 6240 & 3644 & 3323 & 1592 & 1055 & 640 & 1055 & 748 & 141522 \\
\hline TOTAL ACUM. & 1132 & 2337 & 2861 & 3956 & 5869 & 8773 & 11894 & 15096 & 21678 & 36806 & 57936 & 77246 & 90400 & 104089 & 9113955 & 123225 & 129465 & 133109 & 136432 & 138024 & \begin{tabular}{|l|}
39079 \\
\end{tabular} & 139719 & 140774 & 141522 & 141522 \\
\hline
\end{tabular}




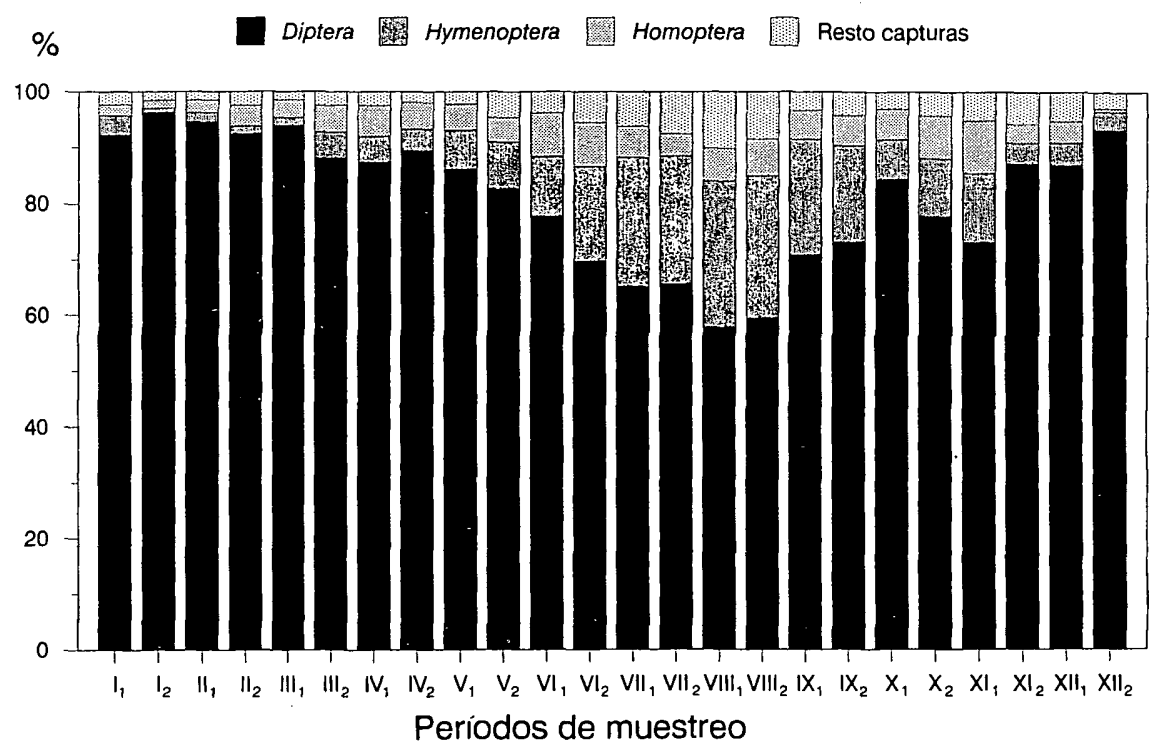

Fig. 5. Porcentaje sobre el total de los cuatro órdenes de insectos mayoritarios a lo largo de los períodos de muestreo.

Percentages over the total of the four main insect orders through the sampled years.

interrumpir su vuelo la red central de la trampa Malaise. Por otro lado, los himenópteros aumentan su frecuencia progresivamente desde la primavera hasta finales de verano; los valores de frecuencia anual oscilan entre 0,8$26,6 \%$. Durante el período estival se observa un incremento de himenópteros respecto a los dípteros (Fig. 5) que no es debido, a tenor de los datos, a un aumento de capturas de himenópteros, sino que se debe a un descenso significativo de las capturas de los dípteros, en contraposición a un mantenimiento o un descenso más gradual de capturas de los himenópteros. El descenso en los himenópteros durante la primera quincena de julio (fig. 6b) así como la relativa brusquedad en la disminución de ejemplares capturados de dípteros (fig. 6a), puede deberse a que durante este período las lluvias fueron más abundantes. Esta apreciación también puede extrapolarse a aquellos otros órdenes de insectos con relativa presencia numérica (fig. 7 y 8). No obstante en el caso de los lepidópteros este efecto está más acentuado al coincidir con el límite de la presencia de adultos invernales y temporales (fig. 7c), y en el caso de los coleópteros (Fig. 7a) por el tipo de capturas obtenidas (mayoritariamente ejemplares muy pequeños). Finalmente, el tercer orden mayoritario, los homópteros (Fig. 5), presentes también durante todo el año muestreado, presentan una frecuencia de aparición que oscila entre el 0,6- 

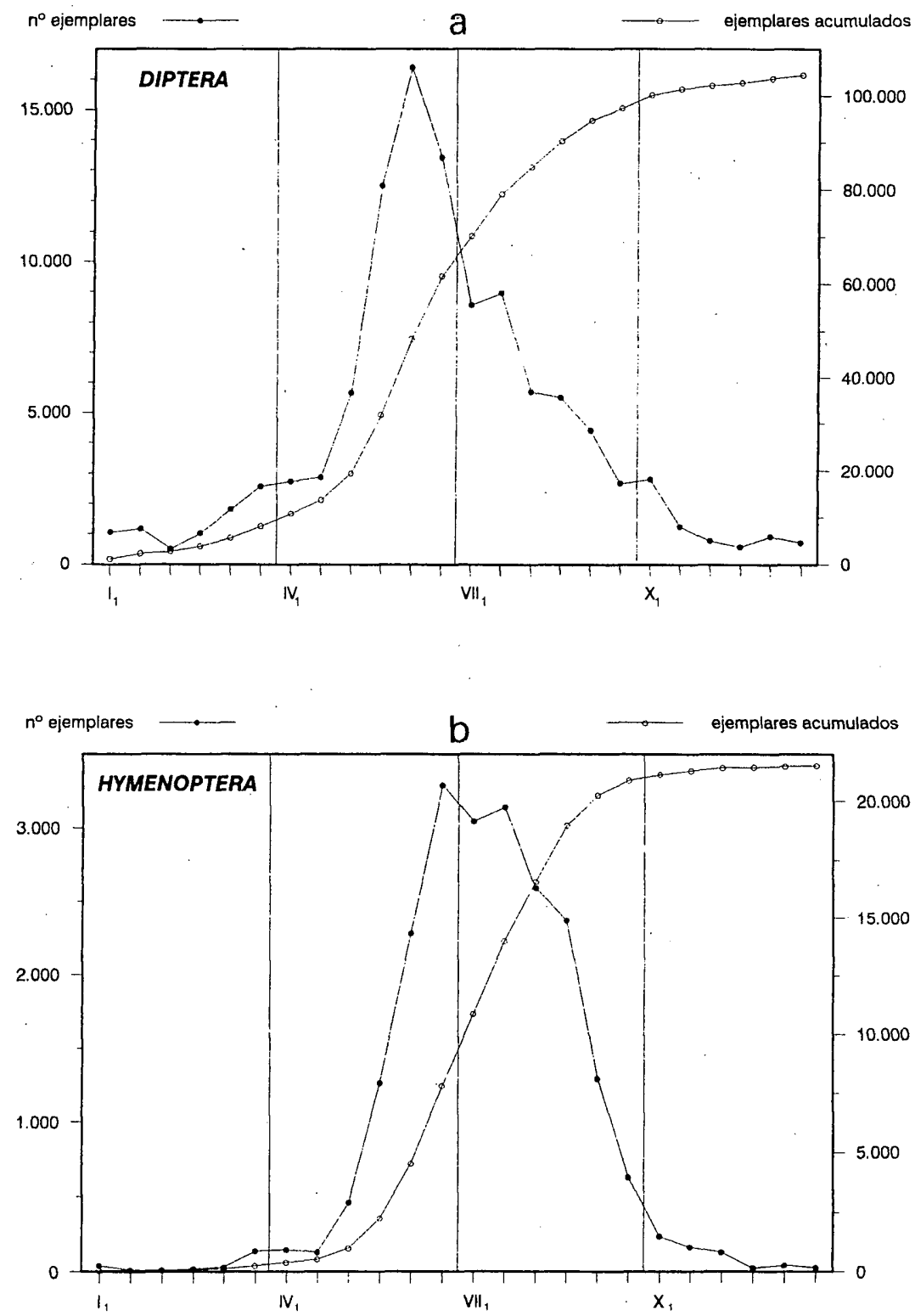

Fig. 6. Evolución de las capturas de los dos órdenes de insectos más abundantes. Evolution of the captures of the more abundant insect orders. 
$7,9 \%$. Cabe resaltar la homogeneidad porcentual de este orden en la mayoría del año, aunque no sucede así en el número de capturas, truncado solamente en el período invernal menos térmico $\left(\mathrm{XII}^{2}-\mathrm{II}^{2}\right)$.

Por lo que hace referencia a los órdenes de insectos menos abundantes observamos en principio dos modelos: los que presentan el máximo relativo durante el segundo trimestre anual (primavera), al que se adscriben también los dípteros (Fig. 6a), y aquellos en los que se encuentra el máximo durante el tercer trimestre (y por tanto son de fenología estival) como ocurre con los himenópteros (Fig. 6b).

Respecto al primer grupo destacan los homópteros (Fig. 7b), los heterópteros (Fig. 7d), los tisanópteros (Fig. 7f), los plecópteros (Fig. 8a), los blatópteros (Fig. 8b) y los mecópteros (Fig. 8c). En relación a los heterópteros, cuyo material ha sido ya estudiado desde un punto de vista específico (GESSÉ \& al. 1994) hemos de destacar que la explosión cuantitativa observada en la segunda quincena de mayo es debida a la captura masiva del mírido Harpocera thoracica, especie propia de fagáceas (robles y encinas). Cualitativamente se obtuvieron 81 especies pertenecientes a 11 familias distintas. Por otro lado todos los blatópteros colectados corresponden a Eclobius pallidus f. chopardi (según indicaciones del Dr. Pardo). El resto del material aún está en estudio.
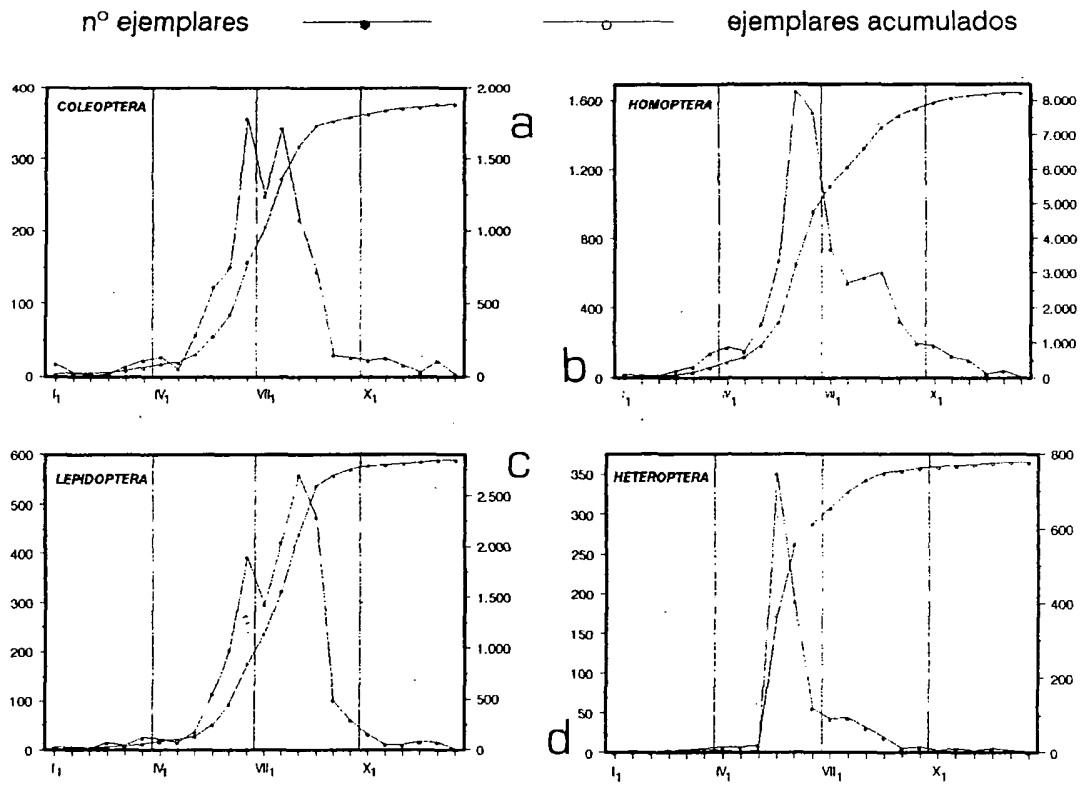

Fig. 7. 
En el segundo grupo de órdenes de insectos, los de preponderancia estival, incluye los coleópteros (Fig. 7a), lepidópteros (Fig. 7c), los psocópteros (Fig. 7e), planipennios (Fig. 7f), dermápteros (Fig. 7h) y ortópteros (Fig. 8d). Destaca el hecho de que la mayoría de los ortópteros capturados (10 especies pertenecientes a tres familias, según indicación del Dr. Olmo) así como los. dermápteros, incluyen individuos poco o nada voladores y que por tanto han accedido al recipiente colector trepando por la trampa. Los psocópteros, que han sido estudiados por el Dr. Baz, incluyen 17 especies pertenecientes a 8 familias distintas. Los planipennios, examinados por la Dra. Díaz-Aranda, representan un total de 32 especies pertenecientes a tres familias distintas. En referencia a los lepidópteros la mayoría de ejemplares capturados son microlepidópteros. Los coleópteros, a pesar de ser el orden de insectos más amplio, se obtienen en un bajo porcentaje, debido a la tendencia que tienen estos insectos a dejarse caer al suelo y escapar cuando encuentran un obstáculo durante el vuelo (MATHEWS \& MATHEWS, 1970); ello viene apoyado, atendiendo al tipo de capturas obtenido, en el hecho que los individuos colectados son de tamaño pequeño o muy pequeño por lo que hay todo un conjunto de grupos que no se consiguen colectar con este tipo de trampa. Una solución a ello, y para aumentar la eficiencia del muestreo en su conjunto, consiste en colocar debajo de la red central de la trampa Malaise una bandeja colectora con algún líquido no atrayente que impida la pérdida del material atrapado, aspecto que no ha sido considerado en este estudio.
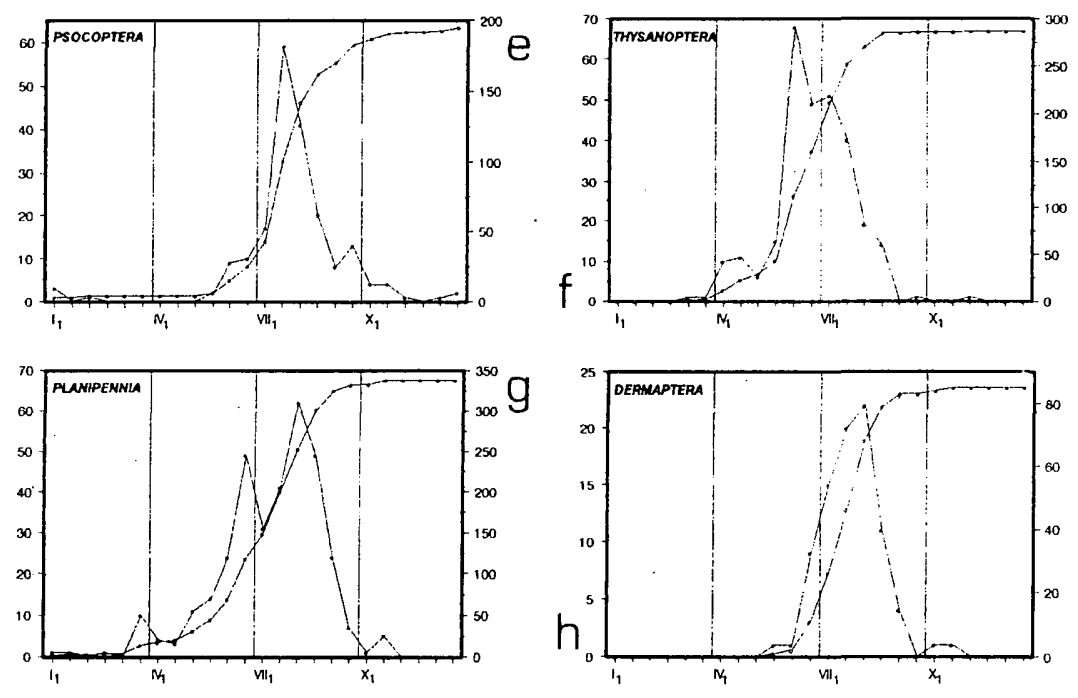

Fig. 7. Evolución de las capturas de ocho órdenes de insectos. Evolution of the captures of eight insect orders. 
PIRINEOS 147-148
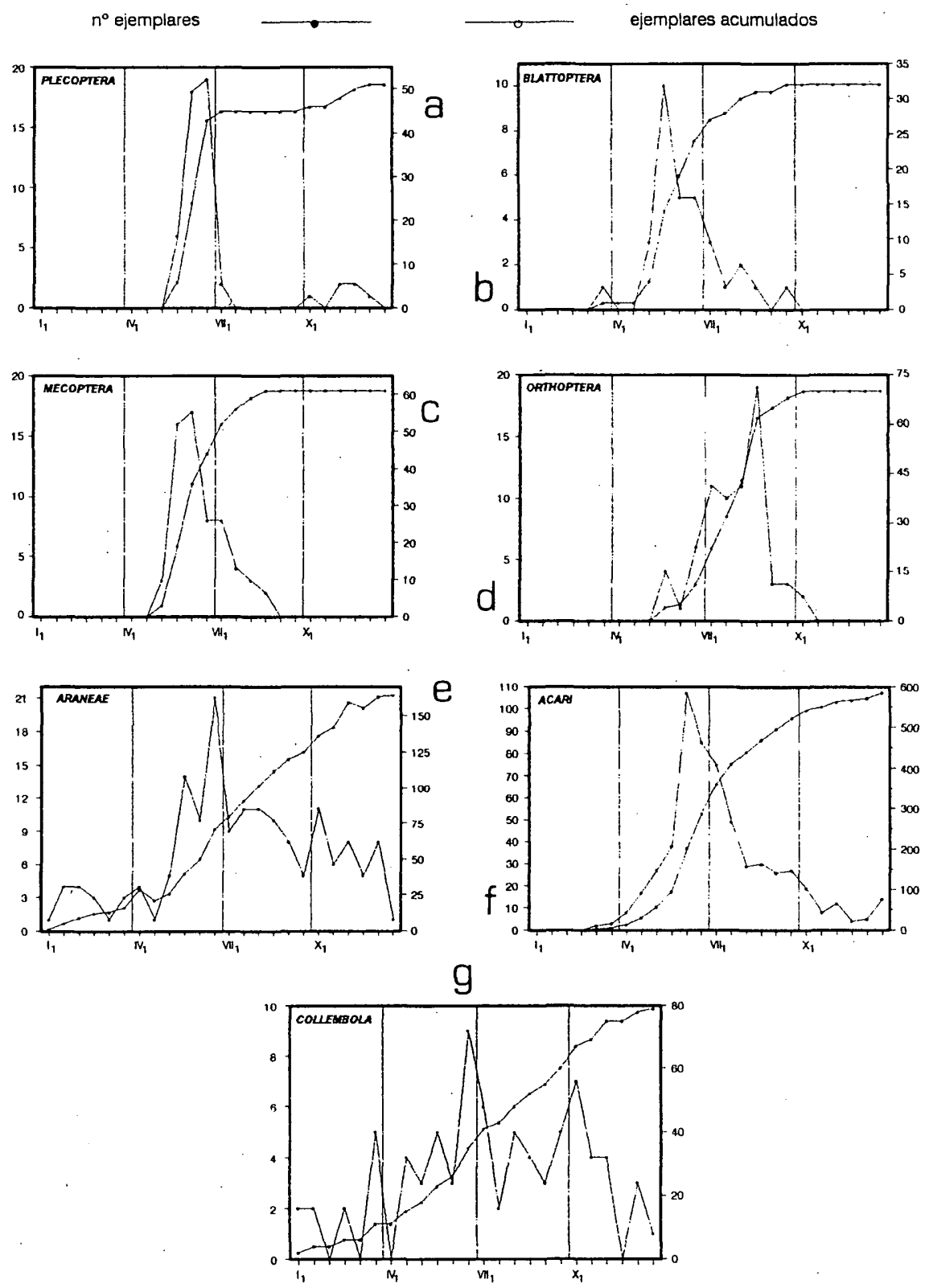

Fig. 8.- Evolución de las capturas de cuatro órdenes de insectos y de los restantes grupos de artrópodos colectados.

Evolution of the number of captured insects of four orders and of the remaining arthropoda collected. 
En relación con los otros órdenes de insectos, el escaso material obtenido no nos permite profundizar sobre ellos. Sólo destacaremos que el estudio de los tricópteros ha puesto de manifíesto la presencia de 5 especies distintas a partir tan sólo de 8 ejemplares, según indicaciones del Dr. González.

En cuanto al resto de órdenes colectados hemos de destacar ciertas particularidades. La presencia de arañas en el recipiente colector se debe fundamentalmente al carácter depredador que tienen los ejemplares capturados; de ello se desprende que debieron caer al recipiente colector mientras perseguían una presa que seguramente tuvo el mismo destino. Hemos observado, por otro lado, que alrededor de la luz del recipiente colector existe mucho movimiento de los individuos durante los períodos de actividad, lo cual de seguro ha de atraer a las arañas que se introduzcan en la trampa. Ello hace que pese a presentarse durante todo el año, la evolución de las capturas no siga ningún patrón "gaussiano" (Fig. 8e). Lo mismo puede decirse de los colémbolos (Fig. 8g) aunque por otras causas. En efecto, la presencia de estos hexápodos en el recipiente colector, teniendo en cuenta el tamaño reducido de los individuos y la movilidad de los mismos, sólo puede explicarse por su capacidad de trepar. Ello se pone de manifiesto al estudiar el matérial colectado que pertenece mayoritariamente a Entomobrya, género en el que se encuentran especies marcadas por su preferencia arbustiva (según Mateos, com. pers.). Por otro lado, parece indicar, a tenor de los resultados obtenidos, que el número de colémbolos en la zona estudiada ha de ser elevado, pues la luz del recipiente colector es muy pequeña en comparación con el tamaño de la trampa. Finalmente, la presencia de ácaros es debida al carácter forésico de este grupo de artrópodos. Ello se pone de manifiesto al observar la similitud entre la curva de abundancia de los ácaros (Fig. 8f) y la curva de capturas de los distintos períodos de muestreo (Fig. 4). Éstos han de haber caído a la trampa transportados por otros insectos principalmente.

\section{Conclusión}

Para concluir, a pesar de no ser un sistema de captura idóneo para la mayoría de los órdenes obtenidos, los datos proporcionados por esta trampa nos reflejan claramente la relación existente entre las condiciones climáticas, la biología general de cada orden y su fenología en un área pirenaica. Así, destaca la presencia del mayor número de ejemplares durante un período de tiempo corto, desplazado especialmente a finales de la primavera. Nuevos datos aparecerán, sin duda, al concluir los estudios cualitativos del material colectado. 
Agradecimientos. Hemos de agradecer muy sinceramente a la familia Mirabet-Gelabert, de la casa Miqueldolça de Santa Coloma, la autorización para colocar la trampa Malaise en su propiedad. Igualmente, agradecemos al D. Toni López y Da. Encarna Carmona, del «Cos de Guardes de Caça i Pesca del Govern Andorra», por haber realizado la recolección periódica de las muestras y el seguimiento y conservación de la trampa durante todo el período de estudio. También agradecemos al Sr. Jordi Guillemet, secretario del Centre de Barcelona del «Institut d'Estudis Andorrans», la tramitación de los datos climatológicos incluidos en este trabajo. Y agradecemos a Javier Blasco Zumeta la información referida a los trabajos que han sido publicados de Pina de Ebro (Zaragoza) a partir dél material colectado en trampa Malaise.

Por otro lado, queremos dar las gracias a nuestros colegas entomólogos Dr. A. Baz y a la Dra. Díaz-Aranda (Universidad de Alcalá), Dr. J. R. Pardo (Universidad de Castilla-La:Mancha), -Dr. J. M. Olmo (Universidad de Barcelona), Dr. M. González (Universidad de Santiago de Compostela) y Dr. E. Mateos (Universidad de Barcelona) por su colaboración en la idẹtificación del material recolectado referido en este estudio.

\section{Referencias}

ASKEW, R.R. (1991): Some Chalcididae (Hym., Chalcidoidea) from Spain with description of a new species of Brachymeria Westwood. Eos, 67: 131133.

ASKEW, R.R. (1993): Two new european species of Ormyrus (Hym., Ormyridae). Entomologist's Monthly Magazine, 130: 87-93.

ASKEW, R.R. (1994): Some Pteromalidae (Hym., Chalcidoidea) from Monegros, with descriptions of four new species. Eos, 69: 75-82.

CARLES-TOLRÁ, M. (1992a): Oldenbergiella pappi sp. n., a new heleomyzid species from Spain (Insecta, Diptera: Heleomyzidae). Reichenbachia, 29: 195-197.

CARLES-TOLRÁ, M. (1992b): Meoneura flavella sp. n., a new carnid species from Spain (Insecta, Diptera: Carnidae). Reichenbachia, 29: 194.

CARLES-TOLRÁ, M. (1993a): Trixoscelis curvata sp. n.: a new trixoscelidid species from Spain (Diptera). Ann. Naturhist. Mus. Wien., 94/95 B: 491-494.

CARLES-TOLRÁ, M. (1993b): A new Trixoscelis species and other Material from Spain (Diptera: Trixoscelididae). Stuttgarter Beitr. Naturk. A, 501 (4): 1-4.

DARLING, D.C. \& PACKER, L. (1988): Effectiveness of Malaise traps in Collecting Hymenoptera: The influence of trap design, mesh size, and 
location. Can. Ent., 120: 787-790.

DELÉCOLLE, J.C. \& RIEB J.P. (1993): Contribution à l'étude des Cératopogonidés d'Espagne. Description de Forcipomyia (Thyridomyia) blascoi n. sp. (Diptera, Nematocera). Nouv. Revue Ent. (N.S.), 10(2): 109-120.

GARRIDO-TORRES, A. \& NIEVES-ALDREY, J.L. (1992a): Estructura y dinámica de una taxocenosis de Pteromalidae (Hym., Chalcidoidea) en el sector medio de la sierra de Guadarrama. Eos, 68 (1): 29-49.

GARRIDO-TORRES, A. \& NIEVES-ALDREY, J.L. (1992b): Nuevas citas de Pteromalidae para España (Hymenoptera, Chalcidoidea) colectados con trampas Malaise. Bol. Soc. Port. Entom., supl. 3, Actas do V Congreso Ibérico de Entomologia: 501-511.

GEIGER, W. (1993): Rediscovery of Dicranomyia (Glochina) mediterranea Lackschewitz \& Pagast, 1942 (Diptera: Limoniidae) in Spain, and neotype designation. Bull. Soc. Entomol. Suisse, 66: 9-13.

GESSÉ, F., GOULA, M. \& PUJADE, J. (1994): Estudi dels heteròpters (Insecta, Heteroptera) capturats amb trampa Malaise a Santa Coloma (Andorra). Sec. Conj. Ent. ICHN-SCL, 8: 61-80.

KRZELJ, S. (1969): Insectes recoltés au piège Malaise aà Peyresq (BassesAlpes). Entomops, 14: 183-196.

MOHRING, W. \& BLASCO-ZUMETA, J. (1992): New Sciarid flies (Diptera, Sciaridae) from the Monegros region (Zaragoza, Spain). Misc. Zool., 16: 93104.

NIEVES-ALDREY, J.L. \& REY del CASTILLO, C. (1991): Ensayo preliminar sobre la captura de insectos por medio de una trampa "Malaise" en la sierra de Guadarrama (España) con especial referencia. a los himenópteros. Ecología, 5: 383-403.

MATHEWS, R.W. \& MATHEWS, J.R. (1970): The Malaise trap. Its utility and potential for sampling insects populations. Mich. Entomol., 4: 117-122.

NOYES, J.S. (1989a): The diversity of Hymenoptera in the tropics with special reference to Parasitica in Sulawesi. Entomological Entomology, 14: 197207.

NOYES, J.S. (1989b): A study of five methods of sampling Hymenoptera (Insecta) in a tropical rainforest, with special reference to the Parasitica. Jour. Nat. Hist., 23: 285-289.

RASO-NADAL, (1992): Consideracions a l'entorn del clima d'Andorra. Annals Inst. Est. And. C. Barc., 1991: 207-212.

STEYSKAL, G.C. (1981): A bibliography of the Malaise trap. Proc. Ent. Soc. Wash., 83: 225-229.

TAN, C.L. (1990): The abundance and Diversity of Hymenopterans in Ulu Kinchin, Pahang, Malaysia. Malayan Nature Journal, 43: 278-281. 
PIRINEOS 147-148

TAN, C.L., KHASHIYAH, M.H., AMINAH, I. \& JAYPRAKASH, P. (1990): Hymenopteran Abundance and Diversity from Three Altitudes at Gunung Janing Barat, Endau-Rompin, Maylasia. Proc. Intern. Conf. Tropical Biod., pp. 225-229.

TERESHKIN, A.M. \& SHLYAKHTYONOK, A.S. (1989): An experience in using Malez's traps to study insects. Rev. Zool., 68 (2): 290-292 (en ruso). 